\title{
Actaticas A-G, Cycloartane Triterpenes From Actaea asiatica With Their Antiproliferative Activity
}

OPEN ACCESS

Edited by:

Guigen $L i$,

Texas Tech University, United States

Reviewed by:

Shao-Jiang Song,

Shenyang Pharmaceutical University,

China

George A. O'Doherty,

Northeastern University, United States

*Correspondence:

Haifeng Wu

hfwu@implad.ac.cn

Xi Chen

chenxi@implad.ac.cn

${ }^{\text {t}}$ These authors have contributed equally to this work

Specialty section:

This article was submitted to

Organic Chemistry,

a section of the journal

Frontiers in Chemistry

Received: 23 April 2021

Accepted: 31 May 2021

Published: 29 July 2021

Citation:

Hu M, Zhao D, Xu X, Ma G, Wu H and

Chen X (2021) Actaticas A-G,

Cycloartane Triterpenes From Actaea asiatica With Their

Antiproliferative Activity.

Front. Chem. 9:695456.

doi: 10.3389/fchem.2021.695456

\author{
Meigeng $\mathrm{Hu}^{1,2,3 \dagger}$, Dan Zhao ${ }^{1,2,3 \dagger}$, Xudong $\mathrm{Xu}{ }^{1,2,3}$, Guoxu $\mathrm{Ma}^{1,2,3}$, Haifeng $\mathrm{Wu}^{1,2,3 *}$ and \\ Xi Chen ${ }^{1,2,3 *}$
}

${ }^{1}$ Key Laboratory of Bioactive Substances and Resource Utilization of Chinese Herbal Medicine, Ministry of Education, Beijing Key Laboratory of Innovative Drug Discovery of Traditional Chinese Medicine (Natural Medicine) and Translational Medicine, Institute of Medicinal Plant Development, Peking Union Medical College and Chinese Academy of Medical Sciences, Beijing, China, ${ }^{2}$ Beijing Key Laboratory of Innovative Drug Discovery of Traditional Chinese Medicine (Natural Medicine) and Translational Medicine, Institute of Medicinal Plant Development, Peking Union Medical College and Chinese Academy of Medical Sciences, Beijing, China, ${ }^{3}$ Key Laboratory of Efficacy Evaluation of Chinese Medicine Against Glycolipid Metabolic Disorders, State Administration of Traditional Chinese Medicine, Institute of Medicinal Plant Development, Peking Union Medical College and Chinese Academy of Medical Sciences, Beijing, China

Phytochemical studies on the rhizomes of Actaea asiatica led to the isolation of seven new cycloartane triterpenes, actaticas A-G (1-7). Their structures were determined by NMR, HRESIMS, and chemical analysis. All the isolates were evaluated for their antiproliferative activity against HT-29 and McF-7 cell lines. The results showed that all the compounds displayed cytotoxicity. All compounds showed significant inhibitory effects with $\mathrm{IC}_{50}$ values of $9.2-26.4 \mu \mathrm{M}$.

Keywords: Actaea asiatica, cycloartane triterpenes, antiproliferative activity, HT-29 cell lines, MCF-7 cell lines

\section{INTRODUCTION}

Actaea asiatica $\mathrm{H}$. Hara, a perennial herb belonging to the family Ranunculaceae, is mainly distributed in the southwest and northwest of China. Its roots have been traditionally used among the Tujia folk in Hubei Province for treating headache, sore throat, rheumatic pain, rubella, measles, pertussis, uterine prolapse, and dog bites (Gao et al., 2006a; Gao et al., 2006b; Fan et al., 2007; Gao et al., 2007). Phytochemical studies indicated that the genus Actaea contained cycloartane triterpene glycosides with cytotoxic activities (Kusano et al., 1998; Kusano et al., 1999; Gao et al., 2006b). However, little systematic chemical work on A. asiatica has been carried out so far. In order to find the bioactive constituents from $A$. asiatica, chemical research were carried out, resulting in the isolation of seven new cycloartane triterpene glycosides, namely, actaticas A-G (1-7) (Figure 1). Their structures were determined by spectroscopic analysis and chemical methods. Herein, structural elucidation of compounds 1-7 was reported as well as their cytotoxic activities.

\section{MATERIALS AND METHODS}

\section{General Experimental Procedures}

Optical rotations were obtained on a PerkinElmer 341 digital polarimeter. IR spectra were recorded on Shimadzu FTIR-8400S spectrometers. NMR spectra were obtained with a Bruker AV III 600 NMR spectrometer (chemical shift values are presented as $\delta$ values with TMS as the internal standard). HR-ESIMS spectra were performed on a LTQ-Obitrap XL spectrometer. Preparative HPLC was performed on a Lumtech K-1001 analytic LC equipped with two pumps of K-501, a UV 


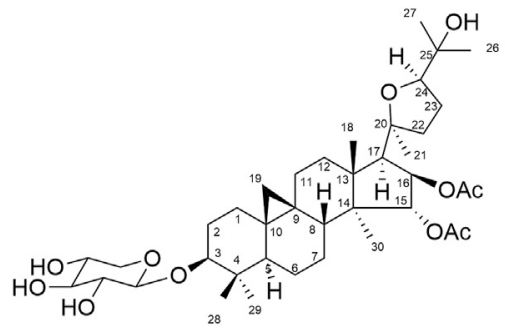

1

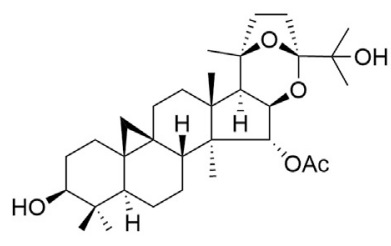

2

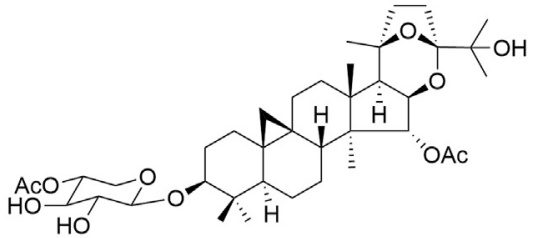

3

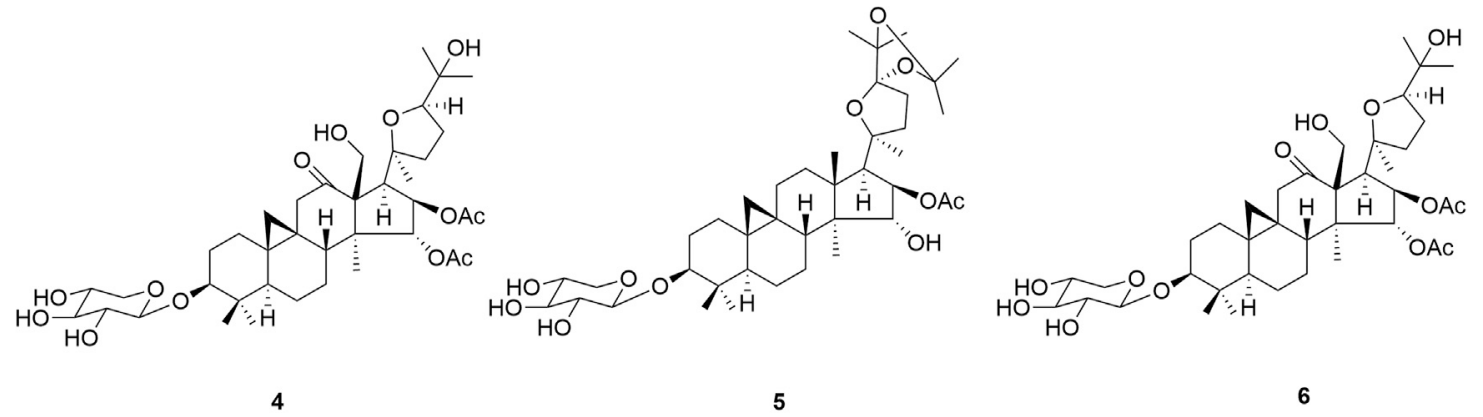

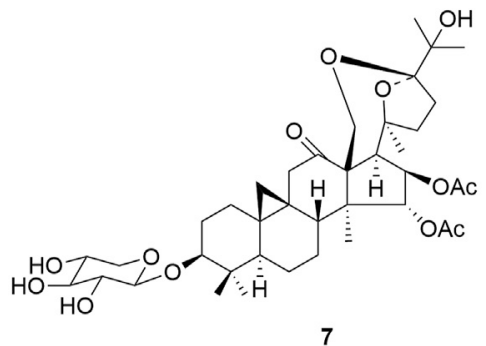

FIGURE 1 | Structures of compounds 1-7.

detector of K-2600, and an YMC Pack $\mathrm{C}_{18}$ column $(250 \times 10 \mathrm{~mm}$, i.d., $5 \mu \mathrm{m}$, YMC Co. Ltd., Japan) eluted with $\mathrm{CH}_{3} \mathrm{OH}-\mathrm{H}_{2} \mathrm{O}$ at a flow rate of $2 \mathrm{ml} / \mathrm{min}$. $\mathrm{C}_{18}$ reversed-phase silica gel $(40-63 \mu \mathrm{m}$, Merk, Darmstadt, Germany), MCI gel (CHP 20P, 75-150 $\mu \mathrm{m}$, Mitsubishi Chemical Corporation, Tokyo, Japan), and silica gel (100-200 mesh, Qingdao Marine Chemical plant, Qingdao, the People's Republic of China) were used for column chromatography. Pre-coated silica gel GF254 plates (Zhi Fu Huang Wu Pilot Plant of Silica Gel Development, Yantai, the People's Republic of China) were used for TLC. All solvents used were of analytical grade (Beijing Chemical Works).

\section{Plant Material}

The plants of $A$. asiatica were collected at Jinfuo Mountain in Chongqing province, the People's Republic of China, in November 2016, and were authenticated by Professor Sirong Yi. The voucher specimen (CS161108) has been deposited at the Institute of Medicinal Plant Development, Chinese Academy of Medical Sciences.

\section{Extraction and Isolation}

The air-dried powdered rhizomes A. asiatica $(6.8 \mathrm{~kg})$ was extracted with $95 \% \mathrm{EtOH}(20 \mathrm{~L})$ three times (each time for $2 \mathrm{~h}$ ). Removal of the $\mathrm{EtOH}$ under reduced pressure yielded the extract $(879 \mathrm{~g})$. The residue was suspended in $\mathrm{H}_{2} \mathrm{O}(1.5 \mathrm{~L})$ and partitioned with petroleum ether $(3 \times 1 \mathrm{~L})$, EtOAc $(3 \times 1 \mathrm{~L})$, acetone $(3 \times 1 \mathrm{~L})$, and $n$ - $\mathrm{BuOH}(3 \times 1 \mathrm{~L})$ successively. The EtOAc fraction $(510 \mathrm{~g})$ was subjected to CC over silica gel $(100-200$ mesh, $8 \times 100 \mathrm{~cm})$ eluting with a stepwise gradient of $\mathrm{CH}_{2} \mathrm{Cl}_{2}-\mathrm{MeOH}$ (from 1:0 to 0:1) to afford six fractions A-F. Fraction B (29.4 g) was subjected to MCI column chromatography $(4 \times 80 \mathrm{~cm})$ elution with $\mathrm{MeOH}-\mathrm{H}_{2} \mathrm{O}(40$ : $60 ; 60: 40 ; 70: 30 ; 80: 20 ; 100: 0, \mathrm{v} / \mathrm{v})$ giving five subfractions (Fr. B1-B5). Subfraction B3 (911 mg) was chromatographed by semi-preparative HPLC using acetonitrile- $\mathrm{H}_{2} \mathrm{O}(75: 25$, v/v) to yield compound $1\left(9.4 \mathrm{mg}, t_{\mathrm{R}}=26.3 \mathrm{~min}\right)$ and $7\left(7.2 \mathrm{mg}, t_{\mathrm{R}}=\right.$ $29.5 \mathrm{~min}$ ). Subfraction B4 (503 mg) was purified through preparative HPLC elution using an acetonitrile- $\mathrm{H}_{2} \mathrm{O}$ (65: 35, $\mathrm{v} / \mathrm{v})$ system to give compound $2\left(12.1 \mathrm{mg}, t_{\mathrm{R}}=23.0 \mathrm{~min}\right)$. 
TABLE 1 | ${ }^{1} \mathrm{H}$ NMR Spectroscopic Data $\left(600 \mathrm{MHz}\right.$, in pyridine- $\left.d_{5}\right)$ for compounds 1-7.

No

\begin{tabular}{|c|c|c|c|c|c|c|}
\hline \multicolumn{7}{|c|}{ Compounds } \\
\hline 1 & 2 & 3 & 4 & 5 & 6 & 7 \\
\hline 1.09 m; 1.52 m & 1.22 m; 1.58 m & 1.20 m; 1.58 m & $1.10 \mathrm{~m} ; 1.35 \mathrm{~m}$ & $1.01 \mathrm{~m} ; 1.52 \mathrm{~m}$ & $1.06 \mathrm{~m} ; 1.49 \mathrm{~m}$ & 1.24 m; 1.37 m \\
\hline 1.92 m; 2.34 m & $1.83 \mathrm{~m} ; 2.40 \mathrm{~m}$ & 1.96 m; 2.25 m & 1.90 m; 2.35 m & 1.92 m; 2.34 m & 1.90 m; 2.34 m & 1.12 m; 2.26 m \\
\hline 3.47dd (12.0, 3.6) & 3.52 dd $(12.0,4.2)$ & 3.36 dd $(11.4,3.0)$ & 3.47 dd $(12.0,3.6)$ & 3.49 dd $(12.0,3.6)$ & 3.48 dd $(12.0,3.6)$ & $3.58 \mathrm{dd}(12.0,3.6)$ \\
\hline $1.27 \mathrm{~m}$ & $1.29, \mathrm{~m}$ & $1.28, \mathrm{~m}$ & $1.20 \mathrm{~m}$ & $1.27 \mathrm{~m}$ & $1.24, \mathrm{~m}$ & $1.28 \mathrm{~m}$ \\
\hline $\begin{array}{c}0.59 \text { q (12.0); } \\
1.38 \mathrm{~m}\end{array}$ & $0.71 \mathrm{q}(6.6)$ & $0.72 \mathrm{~m} ; 1.41 \mathrm{~m}$ & 0.52 q (12.0); 1.38 m & $\begin{array}{c}0.57 \text { q (12.0); } \\
1.33 \mathrm{~m}\end{array}$ & $\begin{array}{c}0.63 \text { q (12.0); } \\
1.38 \mathrm{~m}\end{array}$ & 0.84 m; 1.42 m \\
\hline $\begin{array}{c}1.06 \mathrm{~m} ; 1.31 \mathrm{~m} \\
1.73, \mathrm{~m}\end{array}$ & $\begin{array}{c}1.09 \mathrm{~m} ; 2.09 \mathrm{~m} \\
1.77 \mathrm{dd}(4.8)\end{array}$ & $\begin{array}{c}2.02 \mathrm{~m} ; 1.08 \mathrm{~m} \\
1.72, \mathrm{~m}\end{array}$ & $\begin{array}{c}1.10 \mathrm{~m} ; 1.29 \mathrm{~m} \\
1.63 \mathrm{~m}\end{array}$ & $\begin{array}{c}1.05 \mathrm{~m} ; 1.29, \mathrm{~m} \\
1.72, \mathrm{~m}\end{array}$ & $\begin{array}{c}1.06 \mathrm{~m} ; 1.38, \mathrm{~m} \\
1.77 \mathrm{~m}\end{array}$ & $\begin{array}{c}1.21 \mathrm{~m} ; 1.39 \mathrm{~m} \\
1.78 \mathrm{~m}\end{array}$ \\
\hline $2.03 \mathrm{~m} ; 1.10, \mathrm{~m}$ & $2.02 \mathrm{~m} ; 1.21 \mathrm{~m}$ & $2.00 \mathrm{~m} ; 1.08 \mathrm{~m}$ & $\begin{array}{l}2.04 \text { d (10.8); } \\
1.98 \text { d (10.8) }\end{array}$ & $2.02 \mathrm{~m} ; 1.10 \mathrm{~m}$ & $\begin{array}{l}2.43 \text { d (10.8); } \\
1.99 \text { d (10.8) }\end{array}$ & $\begin{array}{l}2.25 \text { d (10.8); } \\
1.92 \text { d (10.8) }\end{array}$ \\
\hline $\begin{array}{c}2.37 \mathrm{~m} ; 1.76, \mathrm{~m} \\
5.49 \mathrm{~d}(4.8)\end{array}$ & $\begin{array}{c}1.55 \mathrm{~m} ; 2.30 \mathrm{~m} \\
5.70 \mathrm{~d}(3.0)\end{array}$ & $\begin{array}{c}2.32 \mathrm{~m} ; 1.71 \mathrm{~m} \\
5.67 \mathrm{~d}(3.0)\end{array}$ & $5.88 \mathrm{~d}(4.8)$ & $\begin{array}{c}2.36 \mathrm{~m} ; 1.76 \mathrm{~m} \\
5.54 \mathrm{~d}(4.8)\end{array}$ & $1.47 \mathrm{~m} ; 2.50 \mathrm{~d}(8.4)$ & $5.50 \mathrm{~d}(4.8)$ \\
\hline $5.82 \mathrm{dd}(4.8,1.2)$ & 4.47 q (3.0) & $4.30 \mathrm{dd}(8.4,3.0)$ & $5.90 \mathrm{dd}(9.6,4.8)$ & 5.95 dd $(9.6,4.8)$ & $5.47 \mathrm{t}(8.4)$ & $5.78 \mathrm{dd}(9.6,4.8)$ \\
\hline $2.76, \mathrm{~s}$ & $2.00, \mathrm{~m}$ & $2.00 \mathrm{~m}$ & $2.01 \mathrm{~m}$ & $2.80 \mathrm{~d}(10.2)$ & $2.92 \mathrm{~d}(9.6)$ & 3.02 t (8.4) \\
\hline $3.29, \mathrm{~s}$ & $1.72, \mathrm{~m}$ & $1.89, \mathrm{~s}$ & $\begin{array}{l}3.52 \text { d (12.0); } \\
4.18 \text { d (12.0) }\end{array}$ & $1.49 \mathrm{~s}$ & $\begin{array}{l}3.51 \text { d (11.4); } \\
4.03 \text { d (11.4) }\end{array}$ & $\begin{array}{l}3.54 \text { d (11.4); } \\
4.13 \text { d (11.4) }\end{array}$ \\
\hline $\begin{array}{l}0.18 \text { d (4.2); } \\
0.47 \text { d (4.2) }\end{array}$ & $\begin{array}{c}0.53, \text { d (4.2); } \\
0.32 \text { d (4.2) }\end{array}$ & $\begin{array}{l}0.21 \text { d (4.2); } \\
0.42 \mathrm{~d}(4.2)\end{array}$ & $\begin{array}{l}0.18 \text { d (4.2); } \\
0.39 \text { d (4.2) }\end{array}$ & $\begin{array}{l}0.26 \text { d (4.2); } \\
0.51 \text { d (4.2) }\end{array}$ & $\begin{array}{l}0.12 \text { d (4.2); } \\
0.44 \text { d (4.2) }\end{array}$ & $\begin{array}{l}0.28 \text { d (4.2); } \\
0.41 \text { d (4.2) }\end{array}$ \\
\hline $1.47 \mathrm{~s}$ & $1.61 \mathrm{~s}$ & $1.58 \mathrm{~s}$ & $1.30 \mathrm{~s}$ & $1.74 \mathrm{~s}$ & $1.30 \mathrm{~s}$ & $1.32 \mathrm{~s}$ \\
\hline $2.37 \mathrm{~m} ; 1.76 \mathrm{~m}$ & 2.02 m; 1.78 dd (4.8) & $2.03 \mathrm{~m} ; 1.80 \mathrm{~m}$ & $2.36 \mathrm{~m} ; 1.77 \mathrm{~m}$ & $2.33 \mathrm{~m} ; 1.76 \mathrm{~m}$ & $2.39 \mathrm{~m} ; 1.77 \mathrm{~m}$ & 2.24 m; 1.63 m \\
\hline $\begin{array}{c}2.29 \mathrm{~m} ; 1.90, \mathrm{~m} \\
3.83 \mathrm{t}(7.2)\end{array}$ & $\begin{array}{c}2.02 \mathrm{~m} ; 1.57 \mathrm{~m} \\
-\end{array}$ & $\begin{array}{c}2.02 \mathrm{~m} ; 1.73 \mathrm{~m} \\
-\end{array}$ & $\begin{array}{c}2.18 \mathrm{~m} ; 1.90 \mathrm{~m} \\
3.72 \mathrm{t}(7.2)\end{array}$ & $\begin{array}{c}2.29 \mathrm{~m} ; 1.90 \mathrm{~m} \\
-\end{array}$ & $\begin{array}{c}2.18 \mathrm{~m} ; 1.90 \mathrm{~m} \\
3.72, \mathrm{t}(7.2)\end{array}$ & $\begin{array}{c}2.12 \mathrm{~m} ; 1.92 \mathrm{~m} \\
-\end{array}$ \\
\hline $1.32 \mathrm{~s}$ & $1.66 \mathrm{~s}$ & $1.66 \mathrm{~s}$ & $1.15 \mathrm{~s}$ & $1.33 \mathrm{~s}$ & $1.23 \mathrm{~s}$ & $1.24 \mathrm{~s}$ \\
\hline- & - & - & - & $1.31 \mathrm{~s}$ & - & - \\
\hline $1.36 \mathrm{~s}$ & $1.49, \mathrm{~s}$ & $1.48 \mathrm{~s}$ & $1.55 \mathrm{~s}$ & $1.42 \mathrm{~s}$ & $1.53 \mathrm{~s}$ & $1.51 \mathrm{~s}$ \\
\hline- & - & - & - & $1.45 \mathrm{~s}$ & - & - \\
\hline $1.35 \mathrm{~s}$ & $1.40, \mathrm{~s}$ & $1.38 \mathrm{~s}$ & $1.55 \mathrm{~s}$ & $1.17 \mathrm{~s}$ & $1.55 \mathrm{~s}$ & $1.52 \mathrm{~s}$ \\
\hline $1.02 \mathrm{~s}$ & $1.10, \mathrm{~s}$ & $1.07 \mathrm{~s}$ & $1.00 \mathrm{~s}$ & $1.04 \mathrm{~s}$ & $1.02 \mathrm{~s}$ & $1.01 \mathrm{~s}$ \\
\hline $1.09, \mathrm{~s}$ & $1.23, \mathrm{~s}$ & $1.12 \mathrm{~s}$ & $1.31 \mathrm{~s}$ & $1.25 \mathrm{~s}$ & $1.22 \mathrm{~s}$ & $1.20 \mathrm{~s}$ \\
\hline $2.11 \mathrm{~s}$ & $2.07 \mathrm{~s}$ & $2.06 \mathrm{~s}$ & $1.96 \mathrm{~s}$ & $2.06 \mathrm{~s}$ & - & $2.01 \mathrm{~s}$ \\
\hline $2.13 \mathrm{~s}$ & - & - & $1.91 \mathrm{~s}$ & $2.09 \mathrm{~s}$ & $1.97 \mathrm{~s}$ & $2.03 \mathrm{~s}$ \\
\hline $4.86 \mathrm{~d}(7.2)^{\mathrm{a}}$ & - & $4.81 \mathrm{~d}(7.8)^{\mathrm{b}}$ & $4.85 \mathrm{~d}(7.2)$ & $4.88 d(7.2)$ & $4.86 \mathrm{~d}(7.2)$ & $4.86 \mathrm{~d}(7.2)$ \\
\hline 4.02 t (8.4) & - & $3.97 \mathrm{~m}$ & 4.02 t (8.4) & 4.04 t (8.4) & 4.02 t (8.4) & 4.02 t (8.4) \\
\hline $4.15 \mathrm{t}(9.0)$ & - & $4.18 \mathrm{~m}$ & 4.15 t $(9.0)$ & 4.16 t $(9.0)$ & 4.15 t $(9.0)$ & 4.15 t (9.0) \\
\hline $4.23 \mathrm{~m}$ & - & $4.54 \mathrm{~m}$ & $4.22 \mathrm{~m}$ & $4.23 \mathrm{~m}$ & $4.23 \mathrm{~m}$ & $4.24 \mathrm{~m}$ \\
\hline $\begin{array}{l}3.73 \text { t (8.0); } \\
4.35 \text { dd (4.8) }\end{array}$ & - & 4.34 m; 3.72 m & $3.72 \mathrm{~m} ; 4.35 \mathrm{~m}$ & $3.74 \mathrm{~m} ; 4.37 \mathrm{~m}$ & $3.72 \mathrm{~m} ; 4.35 \mathrm{~m}$ & 3.72 m; 4.35 m \\
\hline- & - & $2.07 \mathrm{~s}$ & - & - & - & - \\
\hline
\end{tabular}

${ }^{a}$ Xylose.

${ }^{b} 4^{\prime}$-acetylxylose.

Fraction D (5.8 g) was loaded on an ODS $\mathrm{C}_{18}$ column $(2 \times$ $80 \mathrm{~cm}$ ) eluted with $\mathrm{MeOH}-\mathrm{H}_{2} \mathrm{O}(40: 60 ; 60: 40 ; 70: 30 ; 80: 20 ; 100$ : $0, \mathrm{v} / \mathrm{v}$ ) to give five subfractions (Fr. D1-D5). Subfraction D3 (503 mg) was chromatographed by semi-preparative HPLC using acetonitrile- $\mathrm{H}_{2} \mathrm{O}(70: 30, \mathrm{v} / \mathrm{v})$ to yield compounds 3 $\left(6.1 \mathrm{mg}, t_{\mathrm{R}}=18.5 \mathrm{~min}\right), 4\left(8.7 \mathrm{mg}, t_{\mathrm{R}}=21.4 \mathrm{~min}\right)$, and $5(7.0$ $\left.\mathrm{mg}, t_{\mathrm{R}}=28.3 \mathrm{~min}\right)$. Fraction $\mathrm{F}(6.7 \mathrm{~g})$ was fractioned on an MCIgel column chromatography eluted with $\mathrm{MeOH}-\mathrm{H}_{2} \mathrm{O}$ (40:60; $60: 40 ; 70: 30 ; 80: 20 ; 100: 0, \mathrm{v} / \mathrm{v}$ ) to give five subfractions (Fr. F1-F5). Subfraction F3 (223 mg) was chromatographed by preparative $\mathrm{HPLC}$ using acetonitrile- $\mathrm{H}_{2} \mathrm{O}(75: 25, \mathrm{v} / \mathrm{v})$ to yield compounds $6\left(5.8 \mathrm{mg}, t_{\mathrm{R}}=22.7 \mathrm{~min}\right)$.

Actatica A (1): $\mathrm{C}_{39} \mathrm{H}_{62} \mathrm{O}_{11}$, white amorphous powder; $[\alpha]$ $20 \mathrm{D}+21.6(c=0.18, \mathrm{MeOH}) ; \mathrm{IR}(\mathrm{KBr}) \nu_{\max }: 3,440,3,397$, $2,924,1,733,1,457,1,044 \mathrm{~cm}^{-1}$; UV $(\mathrm{MeOH}) \lambda_{\max }(\log \varepsilon)$ : $200 \mathrm{~nm}$; for ${ }^{1} \mathrm{H}$ NMR $\left(600 \mathrm{MHz}\right.$, pyridine- $\left.d_{5}\right)$ and ${ }^{13} \mathrm{C}-\mathrm{APT}$ (150 MHz, pyridine- $d_{5}$ ) spectroscopic data, see Tables 1, 2;
HR-ESIMS m/z: 729.4233 (calcd for $\mathrm{C}_{39} \mathrm{H}_{62} \mathrm{O}_{11} \mathrm{Na}[\mathrm{M}+\mathrm{Na}]^{+}$, 729.4184).

Actatica B (2): $\mathrm{C}_{32} \mathrm{H}_{50} \mathrm{O}_{6}$, white amorphous powder; $[\alpha] 20 \mathrm{D}$ $+19.0(c=0.15, \mathrm{MeOH})$; IR $(\mathrm{KBr}) \nu_{\max }: 3,376,2,957,1,738,1,373$, $1,032 \mathrm{~cm}^{-1}$; UV $(\mathrm{MeOH}) \lambda_{\max }(\log \varepsilon): 201 \mathrm{~nm}$; for ${ }^{1} \mathrm{H}$ NMR $\left(600 \mathrm{MHz}\right.$, pyridine- $\left.d_{5}\right)$ and ${ }^{13} \mathrm{C}$-APT $\left(150 \mathrm{MHz}\right.$, pyridine- $\left.d_{5}\right)$ spectroscopic, data see Tables 1, 2; HR-ESIMS m/z: 553.3533 (calcd for $\mathrm{C}_{32} \mathrm{H}_{50} \mathrm{O}_{6} \mathrm{Na}, 553.3500$ ).

Actatica C (3): $\mathrm{C}_{39} \mathrm{H}_{60} \mathrm{O}_{11}$, white amorphous powder; $[\alpha] 20$ $\mathrm{D}+35.1(c=0.31, \mathrm{MeOH})$; IR $(\mathrm{KBr}) \nu_{\max }: 3,493,2,928,1,730$, $1,375,1,044 \mathrm{~cm}^{-1}$; UV (MeOH) $\lambda_{\max }(\log \varepsilon): 201 \mathrm{~nm}$; for ${ }^{1} \mathrm{H}$ NMR $\left(600 \mathrm{MHz}\right.$, pyridine- $\left.d_{5}\right)$ and ${ }^{13} \mathrm{C}-\mathrm{APT}\left(150 \mathrm{MHz}\right.$, pyridine- $\left.d_{5}\right)$ spectroscopic data, see Tables 1, 2; HR-ESIMS m/z: 727.4100 (calcd for $\mathrm{C}_{39} \mathrm{H}_{60} \mathrm{O}_{11} \mathrm{Na},[\mathrm{M}+\mathrm{Na}]^{+}$, 727.4088).

Actatica D (4): $\mathrm{C}_{40} \mathrm{H}_{62} \mathrm{O}_{13}$, white amorphous powder; $[\alpha] 20 \mathrm{D}$ + $22.4(c=0.22, \mathrm{MeOH})$; IR (KBr) $v_{\max }: 3,439,2,934,1,734,1,264$, 1,033, 1,033, $962 \mathrm{~cm}^{-1} ; \lambda_{\max }(\log \varepsilon): 201 \mathrm{~nm}$; for ${ }^{1} \mathrm{H}$ NMR 
TABLE $2 \mid{ }^{13} \mathrm{C}$ NMR Data $\left(150 \mathrm{MHz}\right.$, in pyridine- $\left.d_{5}\right)$ for compounds $\mathbf{1 - 7}\left(\delta_{\mathrm{H}}\right.$ in ppm, $J$ in $\mathrm{Hz}$ ).

\begin{tabular}{|c|c|c|c|c|c|c|c|}
\hline \multirow[t]{2}{*}{ Position } & \multicolumn{7}{|c|}{ Compounds } \\
\hline & 1 & 2 & 3 & 4 & 5 & 6 & 7 \\
\hline 1 & 32.6 & 33.0 & 32.6 & 32.8 & 32.6 & 32.9 & 32.8 \\
\hline 2 & 30.9 & 31.7 & 30.9 & 32.3 & 30.1 & 31.1 & 32.3 \\
\hline 3 & 88.8 & 82.8 & 86.7 & 88.8 & 88.7 & 88.8 & 88.7 \\
\hline 4 & 41.7 & 40.5 & 40.5 & 41.7 & 41.7 & 41.7 & 41.7 \\
\hline 5 & 47.7 & 47.8 & 47.8 & 47.7 & 47.6 & 47.7 & 47.4 \\
\hline 6 & 21.2 & 21.7 & 21.4 & 21.1 & 21.1 & 21.4 & 20.8 \\
\hline 7 & 26.5 & 26.5 & 26.4 & 26.5 & 26.1 & 26.7 & 26.5 \\
\hline 8 & 48.3 & 48.3 & 48.2 & 48.5 & 48.0 & 50.3 & 49.0 \\
\hline 9 & 19.9 & 20.1 & 20.1 & 20.2 & 19.8 & 20.7 & 20.2 \\
\hline 10 & 26.7 & 27.1 & 26.7 & 26.9 & 27.1 & 26.9 & 26.9 \\
\hline 11 & 26.3 & 26.6 & 26.5 & 31.2 & 27.0 & 31.9 & 31.2 \\
\hline 12 & 37.5 & 25.9 & 26.4 & 216.6 & 39.1 & 216.6 & 216.6 \\
\hline 13 & 48.6 & 50.3 & 50.3 & 59.3 & 48.3 & 59.0 & 59.1 \\
\hline 14 & 47.6 & 47.0 & 47.0 & 42.4 & 46.4 & 42.7 & 42.4 \\
\hline 15 & 86.4 & 80.3 & 76.7 & 86.1 & 85.7 & 42.7 & 82.4 \\
\hline 16 & 79.8 & 78.3 & 80.3 & 80.1 & 79.8 & 77.3 & 79.1 \\
\hline 17 & 56.1 & 51.6 & 51.6 & 52.7 & 59.0 & 53.2 & 56.5 \\
\hline 18 & 21.7 & 14.1 & 33.5 & 64.4 & 26.1 & 64.5 & 64.7 \\
\hline 19 & 30.5 & 31.2 & 30.4 & 26.1 & 30.5 & 26.2 & 26.5 \\
\hline 20 & 85.2 & 86.7 & 82.9 & 86.6 & 87.3 & 86.6 & 87.2 \\
\hline 21 & 27.6 & 25.1 & 25.1 & 27.7 & 22.3 & 24.4 & 24.7 \\
\hline 22 & 33.7 & 41.5 & 41.4 & 39.0 & 39.1 & 39.1 & 32.3 \\
\hline 23 & 27.0 & 29.0 & 29.0 & 29.7 & 30.5 & 31.2 & 30.9 \\
\hline 24 & 83.8 & 111.2 & 111.2 & 81.7 & 115.2 & 81.6 & 114.7 \\
\hline 25 & 70.9 & 72.4 & 72.4 & 71.7 & 82.8 & 71.7 & 71.6 \\
\hline 26 & 27.6 & 25.9 & 25.8 & 26.1 & 30.3 & 26.1 & 26.4 \\
\hline 27 & 27.6 & 26.5 & 25.9 & 21.7 & 22.3 & 21.5 & 26.3 \\
\hline 28 & 26.1 & 25.7 & 25.6 & 21.7 & 26.1 & 21.5 & 26.3 \\
\hline 29 & 15.8 & 15.3 & 15.6 & 14.5 & 15.7 & 15.8 & 15.7 \\
\hline 30 & 13.8 & 14.1 & 14.1 & 13.7 & 13.9 & 14.5 & 15.2 \\
\hline \multirow[t]{2}{*}{$15-A c$} & 170.9 & 170.7 & 170.6 & 171.5 & 171.2 & & 171.5 \\
\hline & 21.2 & 22.0 & & 21.5 & 21.7 & & 21.6 \\
\hline \multirow[t]{2}{*}{$16-A c$} & 171.2 & & & 171.1 & 170.2 & 171.8 & 171.1 \\
\hline & 22.0 & & & 21.6 & 21.8 & 24.4 & 21.4 \\
\hline $3-A c$ & & & & & & 76.0 & \\
\hline $1^{\prime}$ & $108.1^{a}$ & & $105.1^{\mathrm{b}}$ & $108.1^{a}$ & $107.9^{a}$ & $108.0^{a}$ & $108.1^{a}$ \\
\hline $2^{\prime}$ & 76.0 & & 71.7 & 76.0 & 76.0 & 76.0 & 76.0 \\
\hline $3^{\prime}$ & 79.1 & & 76.0 & 79.4 & 79.1 & 79.1 & 79.1 \\
\hline $4^{\prime}$ & 71.6 & & 89.0 & 71.1 & 71.7 & 71.7 & 71.7 \\
\hline $5^{\prime}$ & 67.6 & & 67.6 & 67.6 & 67.6 & 67.6 & 67.6 \\
\hline \multirow[t]{2}{*}{$4^{\prime}-A c$} & & & 170.6 & & & & \\
\hline & & & 21.9 & & & & \\
\hline $26^{\prime}$ & & & & & 30.3 & & \\
\hline $27^{\prime}$ & & & & & 27.4 & & \\
\hline
\end{tabular}

axylose.

${ }^{b} 4^{\prime}$-acetylxylose.

(600 MHz, pyridine- $\left.d_{5}\right)$ and ${ }^{13} \mathrm{C}-\mathrm{APT}\left(150 \mathrm{MHz}\right.$, pyridine- $\left.d_{5}\right)$ spectroscopic data, see Tables 1, 2; HR-ESIMS $\mathrm{m} / z$ : 759.3974 (calcd for $\mathrm{C}_{40} \mathrm{H}_{62} \mathrm{O}_{13} \mathrm{Na}[\mathrm{M}+\mathrm{Na}]^{+}, 759.3926$ ).

Actatica E (5): $\mathrm{C}_{42} \mathrm{H}_{66} \mathrm{O}_{12}$, white amorphous powder; $[\alpha] 20 \mathrm{D}$ $+42.7(c=0.35, \mathrm{MeOH}) ; \mathrm{IR}(\mathrm{KBr}) v_{\max }: 3,449,2,935,1,741,1,375$, $1,045 \mathrm{~cm}^{-1} ; \lambda_{\max }(\log \varepsilon): 200 \mathrm{~nm}$; for ${ }^{1} \mathrm{H}$ NMR $(600 \mathrm{MHz}$, pyridine) and ${ }^{13} \mathrm{C}-\mathrm{APT}\left(150 \mathrm{MHz}\right.$, pyridine- $\left.d_{5}\right)$ spectroscopic data, see Tables 1, 2; HR-ESIMS $\mathrm{m} / \mathrm{z}$ : 785.4341 (calcd for $\left.\mathrm{C}_{42} \mathrm{H}_{66} \mathrm{O}_{12} \mathrm{Na}[\mathrm{M}+\mathrm{Na}]^{+}, 785.4341\right)$.

Actatica F (6): $\mathrm{C}_{37} \mathrm{H}_{58} \mathrm{O}_{11}$, white amorphous powder; $[\alpha] 20 \mathrm{D}$ $+23.3(c=0.12, \mathrm{MeOH}) ; \mathrm{IR}(\mathrm{KBr}) v_{\max }: 3,350,2,820,1,710,1,534$, $952 \mathrm{~cm}^{-1} ; \lambda_{\max }(\log \varepsilon): 202 \mathrm{~nm}$; for ${ }^{1} \mathrm{H}$ NMR $(600 \mathrm{MHz}$, pyridine- $d_{5}$ ) and ${ }^{13} \mathrm{C}$-APT (150 MHz, pyridine- $\left.d_{5}\right)$ spectroscopic data, see Tables 1, 2; HR-ESIMS $m / z$ : $701.3904\left([\mathrm{M}+\mathrm{Na}]^{+}\right.$, calcd for $\mathrm{C}_{37} \mathrm{H}_{58} \mathrm{O}_{11} \mathrm{Na}, 701.3926$ ).

Actatica G (7): $\mathrm{C}_{39} \mathrm{H}_{58} \mathrm{O}_{13}$, white amorphous powder; $[\alpha] 20 \mathrm{D}+$ $22.6(c=0.13, \mathrm{MeOH})$; IR $(\mathrm{KBr}) v_{\text {max }}: 3,298,2,781,1,716,1,422$, $950 \mathrm{~cm}^{-1}$; for ${ }^{1} \mathrm{H}$ NMR (600 MHz, pyridine- $d_{5}$ ) and ${ }^{13} \mathrm{C}$-APT $\left(150 \mathrm{MHz}\right.$, pyridine- $\left.d_{5}\right)$ spectroscopic data, see Tables 1, 2; HRESIMS $m / z: 757.3765\left([\mathrm{M}+\mathrm{Na}]^{+}\right.$, calcd for $\mathrm{C}_{39} \mathrm{H}_{58} \mathrm{O}_{13} \mathrm{Na}$, 757.3770).

\section{Hydrolysis of Compounds}

Acid hydrolysis of 1 and 4-7: A solution of compounds 1, 4, 5, 6, and $7(5 \mathrm{mg})$ in $2 \mathrm{M} \mathrm{HCl}(1 \mathrm{ml})$ was heated at reflux for $24 \mathrm{~h}$. The reaction mixture was neutralized with $2 \mathrm{M} \mathrm{NaOH}$ and extracted by partition with EtOAc $(5 \times 1 \mathrm{ml}) .10 \mathrm{ml}$ of water was added to the residue and extracted with $\mathrm{CH}_{2} \mathrm{Cl}_{2}$ three times. Sugars were analyzed by TLC and GC analysis and compared with authentic sample of D-sugars. The spots were visualized by spraying with $\mathrm{EtOH}-\mathrm{H}_{2} \mathrm{SO}_{4}$-anisaldehyde $(9: 0.5: 0.5, \mathrm{v} / \mathrm{v})$, then heated at $150^{\circ} \mathrm{C}$. Furthermore, the absolute configurations of the sugars were determined by gas chromatography according to a method previously described (Ma et al., 2016; Li et al., 2017). Compound 3 was dissolved in $\mathrm{MeOH}(15 \mathrm{ml})$, then $4 \% \mathrm{~K}_{2} \mathrm{CO}_{3}(15 \mathrm{ml})$ was added and each solution was stirred at room temperature overnight. Each solution was neutralized by $10 \% \mathrm{AcOH}$, and extracted with EtOAc $(2 \times 20 \mathrm{ml})$. EtOAc extract after removal of solvent, was dissolved in $\mathrm{MeOH}(10 \mathrm{ml})$ and refluxed with $0.5 \mathrm{~N} \mathrm{HCl}(3 \mathrm{ml})$ for $4 \mathrm{~h}$ (Yin et al., 2010).

\section{Cytotoxic Assay}

The cytotoxicity of compounds 1-7 was evaluated using the MTT procedure with HT-29 and McF-7 cancer cell lines. The cells were incubated in DMEM supplemented with $10 \%$ fetal bovine serum and cultured at a density of $1.2 \times 10^{4} \mathrm{cells} / \mathrm{ml}$ in a 96-well microtiter plate. Five various concentrations of each agent dissolved in dimethyl sulfoxide (DMSO) were then put in the wells. Each concentration was evaluated three times. After incubation under $5 \% \mathrm{CO}_{2}$ at $37^{\circ} \mathrm{C}$ for $48 \mathrm{~h}, 10 \mathrm{ml}$ of MTT ( $4 \mathrm{mg} / \mathrm{ml}$ ) was placed into each well, and the cells were incubated for an additional $4 \mathrm{~h}$. Then, the liquid was taken out, and DMSO $(200 \mathrm{ml})$ was put into the wells. The absorbance was documented with a microplate reader at wave length of $570 \mathrm{~nm}$.

\section{RESULTS AND DISCUSSION}

Compound 1 was obtained as white amorphous powder. Its IR spectrum showed absorptions of hydroxyl group at 3,440 and $3,397 \mathrm{~cm}^{-1}$ and carbonyl at $1,733 \mathrm{~cm}^{-1}$. The HRESIMS spectrum showed a pseudo-molecular ion at $\mathrm{m} / z 729.4233[\mathrm{M}+\mathrm{Na}]^{+}$in the positive ion mode from which in conjunction with NMR data the molecular formula was established as $\mathrm{C}_{39} \mathrm{H}_{62} \mathrm{O}_{11}$, consistent with nine degrees of unsaturation. In the ${ }^{1} \mathrm{H}$ NMR spectrum (Table 1) two cyclopropane-methylene protons as an AX system at $\delta_{\mathrm{H}} 0.18$ and 0.47 (each $1 \mathrm{H}, \mathrm{d}, J=4.0 \mathrm{~Hz}, \mathrm{H}_{2}-19$ ) together with seven tertiary methyl groups at $\delta_{\mathrm{H}} 1.49,1.47,1.32,1.36,1.35,1.02$, and 1.09, indicated a cycloartane triterpenoid structure (Ju et al., 2002a; Mohamed, 2014; Gan et al., 2015; Wu et al., 2017). The ${ }^{1}$ H NMR 


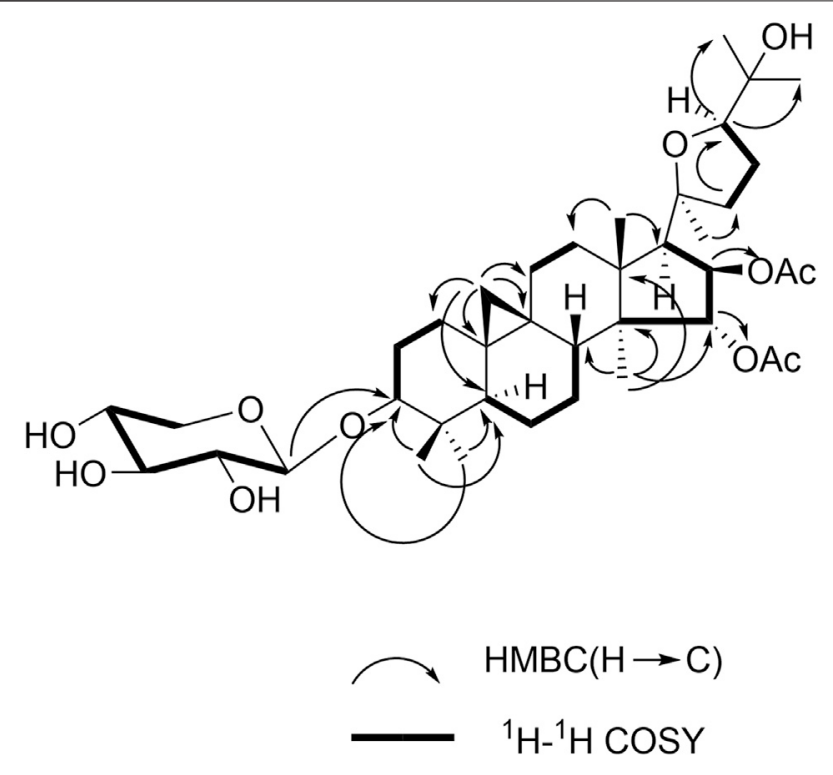

FIGURE 2 | Key $\mathrm{HMBC}$ and ${ }^{1} \mathrm{H}-{ }^{1} \mathrm{H}$ COSY correlations for 1.

spectrum also showed two oxygenated proton signals at $\delta_{\mathrm{H}} 5.49(\mathrm{~d}$, $J=4.8 \mathrm{~Hz})$ and $\delta_{\mathrm{H}} 5.82(\mathrm{~d}, J=4.8 \mathrm{~Hz})$, indicating two acetyl groups at $\mathrm{C}-15$ and $\mathrm{C}-16$. Except for sugar carbons, the ${ }^{13} \mathrm{C}-\mathrm{NMR}$ spectrum (Table 2) of $\mathbf{1}$ displayed 39 carbon resonances including methylene carbon of cyclopropane ring at $\delta_{\mathrm{C}} 30.5(\mathrm{C}$ 19), an oxymethine carbon at $\delta_{\mathrm{C}} 88.8$ (C-3), an oxygenated quaternary carbon at $\delta_{\mathrm{C}} 85.2(\mathrm{C}-20)$, and an anomeric carbon at $\delta_{\mathrm{C}} 108.1$, together with acetyl signals at $\delta_{\mathrm{C}} 170.9,171.2,21.2$, and 22.0. The ${ }^{1} \mathrm{H}$ and ${ }^{13} \mathrm{C}$ NMR spectroscopic data of 1 confirmed that the compound was a cycloartane triterpene glycoside (Jung et al., 2002; Wu et al., 2017; Wua et al., 2017).

All proton signals were assigned to the corresponding carbons through direct ${ }^{1} \mathrm{H}$ and ${ }^{13} \mathrm{C}$ correlations in the HSQC spectrum. Inspection of the ${ }^{1} \mathrm{H}-{ }^{1} \mathrm{H}$ COSY spectrum showed fragments of $\mathrm{C}-1 /$ C-2/C-3, C-5/C-6/C-7/C-8, C-11/C-12, C-15/C-16/C-17, and C22/C-23/C-24. In the HMBC spectrum (Figure 2), the correlations were observed from $\mathrm{H}-28 / 29$ to C-3 and C-5, H-19 to C-1, C-5, C6, C-9, and C-11, and H-18 to C-12 and C-17, H-30 to C-8, C-14, C-16 and C-18, H-21 to C-22, H-22 to C-24, and H-24 to C-26 and C-2 fully confirmed the basic skeleton cycloartane triterpene of compound 1, which was consistent with the above deduction. The acetyl groups were connected with $\mathrm{C}-15$ and $\mathrm{C}-16$ supported by the correlations from $\mathrm{H}-15$ to $\delta_{\mathrm{C}} 170.9$ (the carbonyl carbon of OAc) and $\mathrm{H}-16$ to $\delta_{\mathrm{C}} 171.2$ (the carbonyl carbon of $\mathrm{OAc}$ ). The sugar was connected with $\mathrm{C}-3$ based on the key HMBC correlation between $\mathrm{H}^{-1} 1^{\prime}\left(\delta_{\mathrm{H}} 4.86, \mathrm{~d}, J=7.2 \mathrm{~Hz}\right)$ and $\mathrm{C}-3\left(\delta_{\mathrm{C}} 88.8\right)$, which was identified as D-xylose by TLC in comparison with authentic monosaccharides (visualization with ethanol-5\% $\mathrm{H}_{2} \mathrm{SO}_{4}$ spraying) followed by gas chromatography.

The NOESY experiment and coupling constants established the relative configuration of compound 1 (Figure 3 ), in which correlation of $\mathrm{H}-3 / \mathrm{H}-5$ showed $\alpha$-orientation of $\mathrm{H}-3$. The larger coupling constants $\left({ }^{3} J_{1,2}>7.0 \mathrm{~Hz}\right)$ of the anomeric protons indicated the $\beta$ configuration of the sugar unit. The significant

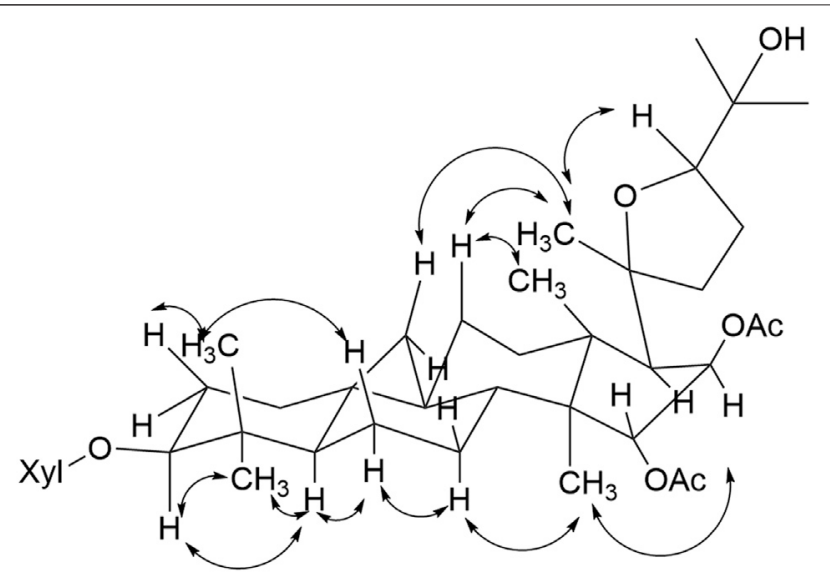

FIGURE 3 | Key NOESY correlations of compound 1.

cross peaks from $\mathrm{H}-15$ to $\mathrm{H}_{3}-18, \mathrm{H}-17 \alpha$ to $\mathrm{Me}-21, \mathrm{H}-16$ to $\mathrm{H}_{3}-30$, and $\mathrm{H}-24$ to $\mathrm{Me}-21$ were observed, which enabled the establishment of OAc- $15 \alpha$ and OAc-16 $\beta$. Until now, all the isolated cycloartane triterpene share the identical absolute configuration with trans $\mathrm{A} / \mathrm{B}$, $\mathrm{B} / \mathrm{C}, \mathrm{C} / \mathrm{D}$ rings. Considering the same cycloartane triterpene skeleton and identical carbon signals at C-20/C-24, compound 1 was established as $20 S$ and $24 R$ configurations (Ju et al., 2002a). Therefore, the structure of the compound was identified as shown and given the trivial name actatica $\mathrm{A}$.

Compound 2 was determined to have the molecular formula $\mathrm{C}_{32} \mathrm{H}_{50} \mathrm{O}_{6}$, by the observation of the ion peak at $\mathrm{m} / z 553.3533$ (calcd for $\mathrm{C}_{32} \mathrm{H}_{50} \mathrm{O}_{6} \mathrm{Na}, 553.3500$ ). The ${ }^{1} \mathrm{H}-\mathrm{NMR}$ spectrum (Tables 1, 2) displayed signals for seven tertiary methyls $\left(\delta_{\mathrm{H}}\right.$ $1.67,1.62,1.54,1.54,1.58,1.08$, and 1.25$)$, two typical signals at $\delta_{\mathrm{H}}$ $0.32(1 \mathrm{H}, \mathrm{d}, J=4.2 \mathrm{~Hz})$ and $0.53(1 \mathrm{H}, \mathrm{d}, J=4.2 \mathrm{~Hz})$ ascribable to a cyclopropane moiety, indicating that 2 might be a cycloartanetype triterpenoid. The ${ }^{13} \mathrm{C}$ NMR spectrum of 2 displayed 32 carbon signals, three signals attributable to oxygen-bearing quaternary carbons at $\delta 82.8,111.2$, and 72.4. The NMR data were similar to the reported one $(20 S, 24 S)-16 \beta, 24 ; 20,24-$ diepoxy-9, 19-cycloeanostane-3 $\beta, 15 \alpha, 18,25$-tetraol-3-O$\beta$ - $\mathrm{D}$-xylopyranoside ( $\mathrm{Mu}$ et al., 2014). The differences were the absence of the sugar at C-3, and the appearance of acetyl group at C-15 in compound 2. In the HMBC spectrum, the correlation observed from $\mathrm{H}-15$ to OAc together with the molecular formula confirmed the deduction above. The $\alpha$ configurations of $H-16$ and $H-17$ were confirmed by the NOESY correlations between $\delta_{H}$ $1.86(H-17)$ and $\delta_{H} 1.23(H 3-30), \delta_{H} 4.47(H-16)$ and $\delta_{H} 2.00$ $(H-17)$. Taken together with the 2D-NMR spectra data, compound 2 was characterized and named actatica $\mathrm{B}$.

Compound 3, which was isolated as a white amorphous powder, was assigned as $\mathrm{C}_{39} \mathrm{H}_{60} \mathrm{O}_{11}$, based on its positive HRESIMS ion at $m / z 727.4100$ (calcd for $\mathrm{C}_{39} \mathrm{H}_{60} \mathrm{O}_{11} \mathrm{Na}$, [M + $\mathrm{Na}]^{+}, 727.4088$ ). The ${ }^{1} \mathrm{H}$ NMR spectrum showed that 3 possesses a cyclopropane ring, seven methyl groups, and an AB-type hydroxymethyl group $\left(\mathrm{H}_{2}-18\right)$. The NMR (Tables 1, 2) spectroscopic data for this compound were analogous to 2, except for the appearance of the anomeric proton at $\delta_{\mathrm{H}} 4.81(\mathrm{~d}$, $J=7.8 \mathrm{~Hz})$ and $\delta_{\mathrm{C}} 105.1,71.7,76.0,89.0,67.6,170.6$, and 22.0. The 
sugar was identified as a $4^{\prime}-\mathrm{O}-\beta_{-}^{-} \mathrm{D}^{-}$xylose after acid hydrolysis. Inspection of the ${ }^{1} \mathrm{H}-{ }^{1} \mathrm{H}$ COSY spectrum showed fragments of $\mathrm{C}-1 /$ C-2/C-3, C-5/C-6/C-7/C-8, C-11/C-12, C-15/C-16/C-17/C-18, and $\mathrm{C}-22 / \mathrm{C}-23$. In the $\mathrm{HMBC}$ spectrum, the correlation from $\mathrm{H}-3(3.36, \mathrm{dd}, J=11.4,3.0 \mathrm{~Hz})$ to the anomeric carbon signal at $\delta_{\mathrm{C}}$ 86.7 supported that the sugar unit was attached to C-3. Thus, the structure of 3 was determined as actatica $\mathrm{C}$.

Compound 4 has a molecular formula of $\mathrm{C}_{39} \mathrm{H}_{60} \mathrm{O}_{13}$ according to the HRESIMS $\left(\mathrm{m} / z\right.$ 759.3974 $[\mathrm{M}+\mathrm{Na}]^{+}$, calcd for $\mathrm{C}_{39} \mathrm{H}_{60} \mathrm{O}_{13} \mathrm{Na}$, 759.3926). Its IR spectrum showed strong hydroxyl $\left(3,439,1,044 \mathrm{~cm}^{-1}\right)$ and carboxyl $\left(1730 \mathrm{~cm}^{-1}\right)$ absorptions. The ${ }^{1} \mathrm{H}$ and ${ }^{13} \mathrm{CNMR}$ spectra indicated that 4 had two acetoxyl groups. Detailed NMR spectral analysis revealed that 4 possessed a cyclopropane ring, six methyl groups, a hydroxymethyl group at C-18, and a D-xylosyl unit at C-3. The ${ }^{1} \mathrm{H}$ and ${ }^{13} \mathrm{C}$ NMR spectra of 4 were similar to those of beesioside $\mathrm{J}$ (Ju et al., 2002b), except for a carbonyl group $(\mathrm{C}=\mathrm{O})$ connected to C-12 of 4, which causes the downfield chemical shift of C-12 $\left(\delta_{\mathrm{C}}\right.$ 216.6). The correlation from $\delta_{\mathrm{H}} 4.54(\mathrm{H}-11)$ to $\delta_{\mathrm{C}} 216.6(\mathrm{C}=\mathrm{O})$ according to the HMBC supported the above result. Therefore, compound 4 was tentatively determined and named actatica D.

Compound 5 has the molecular formula $\mathrm{C}_{42} \mathrm{H}_{66} \mathrm{O}_{12}$ determined by HR-ESIMS $\left(m / z 785.4301\right.$, calcd for $\mathrm{C}_{42} \mathrm{H}_{66} \mathrm{O}_{12} \mathrm{Na}[\mathrm{M}+\mathrm{Na}]^{+}$, 785.4341). In the ${ }^{1} \mathrm{H}$ NMR spectrum (Table 1) two cyclopropane-methylene protons as an AX system at $\delta_{\mathrm{H}} 0.21$ and 0.59 (each $1 \mathrm{H}, \mathrm{d}, J=4.0 \mathrm{~Hz}, \mathrm{H}_{2}-19$ ) together with nine tertiary methyl groups indicated a cycloartane triterpenoid structure. The ${ }^{1} \mathrm{H}$ NMR and ${ }^{13} \mathrm{C}$ APT data for this compound were analogous to 1 , except for the additional NMR signals at $\delta_{\mathrm{C}} 30.3$ and 27.4, and $\delta_{\mathrm{H}}$ $1.31(3 \mathrm{H}, \mathrm{s})$, and $1.45(3 \mathrm{H}, \mathrm{s})$. The differences showed that 5 had one more hydroxyisopropyl group connected at $\mathrm{C}-24$. In the $\mathrm{HMBC}$ spectrum, the correlations from H-24 to C-26, C-26', C-27, and C$27^{\prime}$ confirmed the above deduction. Taken together with the NOESY spectra data, compound 5 was established as $24 R$ configurations. Thus, compound $\mathbf{5}$ was established and named actatica E.

Compound $\mathbf{6}$ was determined to have the molecular formula of $\mathrm{C}_{37} \mathrm{H}_{58} \mathrm{O}_{11}$ based on the ${ }^{13} \mathrm{C}$ APT data and by the HRESIMS ion peak at $m / z 701.3904\left([\mathrm{M}+\mathrm{Na}]^{+}\right.$, calcd for $\left.\mathrm{C}_{37} \mathrm{H}_{58} \mathrm{O}_{11} \mathrm{Na}, 701.3926\right)$. The ${ }^{1} \mathrm{H}-\mathrm{NMR}$ spectrum (Table 1) displayed signals for seven tertiary methyls $(\delta 1.02,1.22,1.23,1.30,1.53$, and 1.55), two typical signals at $\delta 0.12(1 \mathrm{H}, \mathrm{d}, J=4.2 \mathrm{~Hz})$ and $0.44(1 \mathrm{H}, \mathrm{d}, J=4.2 \mathrm{~Hz})$ ascribable to a cyclopropane methylene group, indicating that $\mathbf{6}$ might be a cycloartane-type triterpenoid. Examination of the ${ }^{1} \mathrm{H}$ and ${ }^{13} \mathrm{C}$ APT data (Tables 1, 2) showed the structure of 6 to be similar to 4. The NMR spectrum showed that compound 6 has only one set of acetyl group data. On the basis of ${ }^{1} \mathrm{H}-{ }^{1} \mathrm{H}$ COSY and HSQC and comparison with related 4, all signals were assigned as shown in Tables 1, 2. The correlation from H-16 $\left(\delta_{\mathrm{H}} 5.47\right)$ to acetyl carbon $\left(\delta_{\mathrm{C}} 171.8\right)$ was observed in HMBC spectrum, which means the acetyl group was connected to $\mathrm{C}$ 16. Therefore, compound 6 was clearly determined and named actatica F.

Compound 7 has the molecular formula $\mathrm{C}_{39} \mathrm{H}_{58} \mathrm{O}_{13}$ according to its HRESIMS result $\left(\mathrm{m} / z\right.$ 757.3765 $[\mathrm{M}+\mathrm{Na}]^{+}$, calcd for $\mathrm{C}_{39} \mathrm{H}_{58} \mathrm{O}_{13} \mathrm{Na}, 757.3770$ ). In the ${ }^{1} \mathrm{H}$ NMR spectrum (Table 1) two cyclopropane-methylene protons as an AX system at $\delta_{\mathrm{H}} 0.28$ and $0.41\left(\right.$ each $\left.1 \mathrm{H}, \mathrm{d}, J=4.2 \mathrm{~Hz}, \mathrm{H}_{2}-19\right)$ together with nine tertiary
TABLE 3 | Cytotoxicity of compounds 1-7 against HT-29 and McF-7 cancer cell lines.

\begin{tabular}{lcc}
\hline Compounds & HT-29 $(\boldsymbol{\mu M})$ & McF-7 $(\boldsymbol{\mu M})$ \\
\hline 1 & $10.4 \pm 1.9^{\mathrm{a}}$ & $11.8 \pm 2.6$ \\
2 & $24.6 \pm 2.6$ & $26.4 \pm 1.8$ \\
3 & $21.7 \pm 2.3$ & $23.2 \pm 1.6$ \\
4 & $12.6 \pm 2.8$ & $12.1 \pm 1.5$ \\
5 & $11.0 \pm 1.1$ & $23.9 \pm 3.0$ \\
6 & $17.5 \pm 2.2$ & $12.3 \pm 0.7$ \\
7 & $9.2 \pm 3.0$ & $11.4 \pm 1.9$ \\
$5-F^{b}$ & $3.0 \pm 2.1$ & $2.3 \pm 1.2$
\end{tabular}

${ }^{a}$ Value present mean $\pm S D$ of triplicate experiments.

${ }^{b}$ Positive control substance.

methyl groups indicated a cycloartane triterpenoid structure. The ${ }^{1} \mathrm{H}$ NMR and ${ }^{13} \mathrm{C}$ APT data were closely related to those of beesioside I (Tables 1, 2) (Sakurai et al., 1990). The differences showed that 7 had a carbonyl group attached to C-12, which caused C-12 to move to a lower field, and the chemical shift is greatly increased to $\left.\delta_{\mathrm{C}} 216.6\right)$. The HMBC spectrum shows that $\delta_{\mathrm{H}} 4.54(\mathrm{H}-11)$ is related to $\delta_{\mathrm{C}} 216.6(\mathrm{C}=\mathrm{O})$, confirming the above inference. Moreover, in the NOESY spectrum, correlations were also detected between Me-21/H-22 $\alpha / \mathrm{H}$ $23 \alpha / \mathrm{H}-24 \alpha, \mathrm{H}-22 \alpha / \mathrm{H}-22 \beta, \mathrm{H}-23 \alpha / \mathrm{H}-23 \beta, \mathrm{H}-22 \beta / \mathrm{H}-23 \beta$, and $\mathrm{H}-24 \alpha / \mathrm{Me}-26 / \mathrm{Me}-27$. Considering the same cycloartane triterpene skeletonand identical carbon signals at C-20/C24 , compound 7 enabled a determination of a $20 \mathrm{~S}^{\star}, 24 \mathrm{R}^{\star}$ configuration (Ju et al., 2016). As a result, the structure of 7 was established and named actatica G.

\section{Bioactive Activity}

The cytotoxic of all compounds 1-7 were tested for their inhibitory activity against human HT-29 and McF-7 cancer cell lines using MTT assay. All compounds showed significant inhibitory effects with $\mathrm{IC}_{50}$ values of 9.2-26.4 $\mu \mathrm{M}$ (Table 3). Compound 7, with an oxygen bridge between C-18 and C-24, showed the best potency among the isolated constituents. With a tetrahydrofuran fragment connected by C-20 and C-24, compounds 1 and 4-7 showed better activity than 2 and 3 .

Seven new 9,19-cycloartane glycosides were isolated from the rhizomes of $A$. asiatica $\mathrm{H}$. Hara. Until now, nearly 200 naturally occurring triterpenes with a 9,19-cycloartane have been reported (Su et al., 2016; Hassan et al., 2020). However, compound 5 with one more hydroxy isopropyl group was first isolated from the genus Actaea. All compounds displayed inhibitory activity against human HT-29 and McF-7 cancer cell lines. Further analysis of the data showed that compounds 1 and 4-7 exhibited better protective effect than other compounds, which indicated that the tetrahydrofuran fragment connected by C-20 and C-24 may affect the inhibitory activity regarding HT-29 and McF-7.

\section{DATA AVAILABILITY STATEMENT}

The original contributions presented in the study are included in the article/Supplementary Material; further inquiries can be directed to the corresponding authors. 


\section{AUTHOR CONTRIBUTIONS}

$\mathrm{HW}$ and $\mathrm{XC}$ were responsible for study design. $\mathrm{MH}$ and $\mathrm{DZ}$ were responsible for compound isolation and cytotoxic activity testing. $\mathrm{GM}, \mathrm{XX}, \mathrm{MH}$, and $\mathrm{DZ}$ were responsible for structure elucidation and validation of compound identities. All authors contributed equally to manuscript writing, review, and editing. All authors have read and agreed to the published version of the manuscript.

\section{FUNDING}

This work was financially supported by the Technological Large Platform for Comprehensive Research and Development of New Drugs in the Twelfth Five-Year "Significant New Drugs Created"

\section{REFERENCES}

Fan, Y. S., Yao, Z., Teng, J., Pan, Q., and Duan, H. Q. (2007). Triterpenoids from Actaea Asiatica with Antitumor Activity. Chin. Traditional Herbal Drugs 38 (2), 167-170. doi:10.3321/j.issn:0253-2670.2007.02.003

Gan, L.-S., Zheng, D.-J., Liu, Q., Zhou, J., Zhang, M.-Z., Yao, W., et al. (2015). Eight New Cycloartane Triterpenoids from Beesia Calthifolia with Hepatoprotective Effects against D-Galactosamine Induced L02 Cell Damage. Bioorg. Med. Chem. Lett. 25 (18), 3845-3849. doi:10.1016/j.bmcl.2015.07.070

Gao, J.-C., Zhang, J.-C., Lu, Z.-J., Zhu, G.-Y., Yang, M.-S., and Xiao, P.-G. (2006b). Chemical Constituents of Actaea Asiatica Hara and Their Antiosteoporosis Activities. Biochem. Syst. Ecol. 34 (9), 710-713. doi:10.1016/ j.bse.2006.02.004

Gao, J.-C., Zhang, J.-C., Zhu, G.-Y., Yang, M.-S., and Xiao, P.-G. (2007). Chromones and Indolinone Alkaloids from Actaea Asiatica Hara. Biochem. Syst. Ecol. 35 (7), 467-469. doi:10.1016/j.bse.2007.01.012

Gao, J., Huang, F., Zhang, J., Zhu, G., Yang, M., and Xiao, P. (2006a). Cytotoxic Cycloartane Triterpene Saponins fromActaeaasiatica. J. Nat. Prod. 69 (10), 1500-1502. doi:10.1021/np060113h

Hassan, A. R., Ashour, A., Amen, Y., Nagata, M., El-Toumy, S. A., and Shimizu, K. (2020). A New Cycloartane Triterpene and Other Phytoconstituents from the Aerial Parts of Euphorbia Dendroides. Nat. Product. Res. 1-9. doi:10.1080/ 14786419.2020 .1800693

Ju, J.-H., Liu, D., Lin, G., Xu, X. D., Han, B., Yang, J.-S., et al. (2002a). Beesiosides A-F, Six New Cycloartane Triterpene Glycosides from Beesia Calthaefolia. J. Nat. Prod. 65 (1), 42-47. doi:10.1021/np010293p

Ju, J.-H., Liu, D., Lin, G., Zhang, Y.-M., Yang, J.-S., Lu, Y., et al. (2002b). Beesiosides G, H, and J-N, Seven New Cycloartane Triterpene Glycosides fromBeesiacalthifolia. J. Nat. Prod. 65 (2), 147-152. doi:10.1021/np010294h

Jung, D.-W., Lee, J. M., and Sung, C. K. (2002). Enzyme-linked Immunosorbent Assay for the Determination of 20(S)-protopanaxatriol. Analytica Chim. Acta 462 (2), 157-163. doi:10.1016/S0003-2670(02)00340-9

Kusano, A., Takahira, M., Shibano, M., Miyase, T., Kusano, G., and Bulletin, P. (1999). Studies on the Constituents of Cimicifuga Species. XXVI. Twelve New Cyclolanostanol Glycosides from the Underground Parts of Cimicifuga Simplex WORMSK. Chem. Pharm. Bull. 47 (4), 511-516. doi: $10.1248 / \mathrm{cpb} .47 .511$

Kusano, A., Takahira, M., Shibano, M., Miyase, T., Okuyama, T., and Kusano, G. (1998). ChemInform Abstract: Studies on the Constituents of Cimicifuga Species. Part 22. Structures of Two New Cyclolanostanol Xylosides, Cimiacerosides A and B. ChemInform 5 (48), 1003-1013. doi:10.1002/ chin.199839202

Li, P., Zhu, N., Hu, M., Wu, H., Yu, T., Wu, T., et al. (2017). New Cucurbitane Triterpenoids with Cytotoxic Activities from Hemsleya Penxianensis. Fitoterapia 120, 158-163. doi:10.1016/j.fitote.2017.06.009
Science and Technology Major Projects (No. 2012ZX09301-002001-026) and CAMS Innovation Fund for Medical Sciences, Grant/Award Numbers: CIFS 2019-I2M-1-005).

\section{ACKNOWLEDGMENTS}

Huiling Liu was gratefully acknowledged for her kindness to measuring the NMR spectrum.

\section{SUPPLEMENTARY MATERIAL}

The Supplementary Material for this article can be found online at: https://www.frontiersin.org/articles/10.3389/fchem.2021.695456/ full\#supplementary-material

Ma, G.-X., Feng, W., Sun, Z.-H., Li, P.-F., Zhu, N.-L., Yang, J.-S., et al. (2016). New Stigmastane Type of Steroidal Glycosides from the Roots of Vernonia Cumingiana. J. Carbohydr. Chem. 35 (3), 172-179. doi:10.1080/07328303.2016.1170137

Mohamed, G. A. (2014). New Cytotoxic Cycloartane Triterpene from Cassia Italica Aerial Parts. Nat. Product. Res. 28 (13), 976-983. doi:10.1080/ 14786419.2014.902820

Mu, L.-H., Li, H.-J., Guo, D.-H., Zhao, J.-Y., and Liu, P. (2014). Cycloartane Triterpenes from Beesia Calthaefolia (Maxim.). Fitoterapia 92, 41-45. doi:10.1016/j.fitote.2013.10.005

Sakurai, N., Goto, T., Nagai, M., Inoue, T., and Xiao, P. (1990). Studies on the Constituents of Beesia Calthaefolia, and Souliea Vaginata. III, Breesiodide IV, a Cyclolanostanol Xyloside from the Rhizomes of B. Calthaefolia and S. Vaginata. Heterocycles 30 (2), 897-904. doi:10.3987/COM-89-S78

Su, Y., Chi, W.-C., Wu, L., Wang, Q.-H., and Kuang, H.-X. (2016). Photochemistry and Pharmacology of 9, 19-cyclolanostane Glycosides Isolated from Genus Cimicifuga. Chin. J. Nat. Medicines 14 (10), 721-731. doi:10.1016/S18755364(16)30087-5

Wu, H.-F., Liu, X., Zhu, Y.-D., Zhou, J., Gong, Y.-Y., Ma, G.-X., et al. (2017). A New Cycloartane Triterpenoid Glycoside from Souliea Vaginata. Nat. Product. Res. 31 (21), 2484-2490. doi:10.25135/rnp.10.17.06.10310.1080/ 14786419.2017.1314283

Wua, H., Yang, Z., Wang, Q., Zhub, N., Xub, X., Zou, Q., et al. (2017). A New Cytotoxic Cyclolanostane Triterpenoid Xyloside from Souliea Vaginata. Nat. Prod. Commun. 12 (2), 229-232. doi:10.1177/ 1934578X1701200222

Yin, N., Yan-Li, Z., Chen, J.-C., Lu, L., Qiu, M.-H., and Qiug, C. (2010). Cytotoxic Chemical Constituents from the Roots of Cimicifuga Fetida. J. Nat. Prod. 73 (2), 1192. doi: $10.1021 / \mathrm{np} 9003855$

Conflict of Interest: The authors declare that the research was conducted in the absence of any commercial or financial relationships that could be construed as a potential conflict of interest.

Publisher's Note: All claims expressed in this article are solely those of the authors and do not necessarily represent those of their affiliated organizations, or those of the publisher, the editors and the reviewers. Any product that may be evaluated in this article, or claim that may be made by its manufacturer, is not guaranteed or endorsed by the publisher.

Copyright (C) $2021 \mathrm{Hu}$, Zhao, Xu, Ma, Wu and Chen. This is an open-access article distributed under the terms of the Creative Commons Attribution License (CC BY). The use, distribution or reproduction in other forums is permitted, provided the original author(s) and the copyright owner(s) are credited and that the original publication in this journal is cited, in accordance with accepted academic practice. No use, distribution or reproduction is permitted which does not comply with these terms. 